\title{
Retrospective analysis of single-agent nab-paclitaxel in patients with platinum-resistant non-small cell lung cancer
}

\author{
AYA NAKAYA, TAKAYASU KURATA, TAKASHI YOKOI, YUKI TAKEYASU, MAIKO NIKI, \\ KAYOKO KIBATA, NAOKO SATSUTANI, YOSHITARO TORII, YUICHI KATASHIBA, \\ MAKOTO OGATA, TAKAYUKI MIYARA and SHOSAKU NOMURA
}

First Department of Internal Medicine, Kansai Medical University, Hirakata, Osaka 573-1010, Japan

Received February 25, 2017; Accepted June 2, 2017

DOI: $10.3892 /$ mco.2017.1392

\begin{abstract}
A retrospective study was conducted to investigate the efficacy and toxicity of single-agent nab-paclitaxel in 67 patients with platinum-resistant non-small cell lung cancer in Kansai Medical University Hospital from August 2013 to December 2015. Overall, 25\% of patients experienced disease progression, $48 \%$ exhibited a partial response, $27 \%$ had stable disease and $0 \%$ had a complete response. The median progression-free survival (PFS) time was 4.8 months and the median overall survival time was 18.2 months. There was no statistically significant difference in PFS between patients with non-squamous carcinoma and squamous carcinoma, or between second-line use and post-second-line use. The most common severe adverse event was neutropenia, followed by interstitial lung disease, infection and fatigue. The results revealed that single agent nab-paclitaxel was associated with an acceptable level of toxicity and a favorable response. This regimen has been developed recently, thus it has not been sufficiently evaluated its toxicity and efficacy. Additional studies to evaluate these parameters in non-small cell lung cancer are warranted.
\end{abstract}

\section{Introduction}

Paclitaxel serves an important role in the treatment of advanced non-small cell lung cancer (NSCLC) (1). Use of this drug, however, is greatly limited by its solvent-based formulation with respect to Cremophor $\mathrm{EL}^{\circledR}$ (BASF, Ludwigshafen am Rhein, Germany), which produces serious and dose limiting toxicities. Administration of solvent-based paclitaxel routinely requires long infusion sets (tubing and in-line filters), premedication with steroids and antihistamines to prevent hypersensitivity

Correspondence to: Dr Aya Nakaya, First Department of Internal Medicine, Kansai Medical University, 2-5-1, Shin-Machi, Hirakata, Osaka 573-1010, Japan

E-mail: nakaya1016@yahoo.co.jp

Key words: nab-paclitaxel, non-small cell lung cancer, single-agent, platinum-resistant reactions. Nanoparticle albumin-bound paclitaxel (Nab-PTX) is an albumin-bound formulation of paclitaxel that is readily soluble in saline. This formulation allows the administration of paclitaxel without the use of lipid-based solvents or the need for corticosteroid and antihistamine pre-medication (2).

When administered as In the first-line therapy for patients with NSCLC, nab-PTX, in combination with other platinum reagents, showed has exhibited significant efficacy in improving prognosis as well as in reducing toxicity profiles (3-5). Thus, platinum-based doublet regimens have been widely applied in the first-line treatment of patients with advanced NSCLC.

However, little is currently known regarding the effect of single-agent nab-PTX targeting advanced NSCLC. The phase I/II study of weekly single-agent nab-PTX administered to patients with advanced NSCLC as an initial chemotherapy revealed that the overall response rate (ORR) was 30\%, the median progression free survival (PFS) time was 5 months and the median overall survival (OS) time was 11 months (6). Concerning subsequent treatments, to date, there are only a small number of clinical trials of single-agent nab-PTX.

Hu et al (7) conducted a phase II clinical trial and reported an ORR of $16.1 \%$, a median PFS of 3.5 months and a median OS of 6.8 months. Furthermore, Sakata et al (8) reported an ORR of $31.7 \%$, a median PFS of 4.9 months and a median OS of 13.0 months. However, as these studies are relatively recent (2015-2016) with limited sample sizes, further clinical data are required in order to determine the optimal treatment strategy for the application of single-agent nab-PTX in patients with platinum-resistant NSCLC.

The aim of the current retrospective study was to investigate the efficacy and toxicity of weekly administration of single-agent nab-PTX in patients with platinum-resistant NSCLC.

\section{Patients and methods}

A retrospective study was performed of patients with platinum-resistant NSCLC who were treated with single-agent nab-PTX between August 2013 and December 2015 at the Kansai Medical University Hospital (Osaka, Japan). Eligible patients had a diagnosis of platinum-resistant NSCLC. Exclusion criteria included patients who had not received prior treatment or had been treated with combination chemotherapy. 
Patients were assessed with respect to their clinical stage at the time of diagnosis, according to 7th lung cancer TNM classification (9). This retrospective clinical study was conducted in accordance with the Declaration of Helsinki and the requirements of the local institutional review board of Kansai Medical University Hospital, and received approval from the Ethics Committee of Kansai Medical University.

Treatment. Patients received nab-PTX at a dose of $100 \mathrm{mg} / \mathrm{m}^{2}$ on days 1, 8 and 15 of a 21-day cycle. The drug was administered as an intravenous infusion over $30 \mathrm{~min}$ without corticosteroid or antihistamine pre-medication. The treatment was repeated every four weeks until disease progression was observed or unacceptable toxicity happened.

Study assessments. Patient treatment response was evaluated according to the Response Evaluation Criteria In Solid Tumors (10) via whole-body computerized tomography scans performed every 8-12 weeks until treatment cessation. Toxicity evaluation was based on the Common Toxicity Criteria for Adverse Events version 4.0 (CTCAE v4.0) (http://ctep. cancer.gov/protocolDevelopment/electronic_applications/ctc. htm\#ctc_40).

Statistical analysis. OS was calculated as the duration between the initiation of single-agent nab-PTX treatment and patient mortality the date of the final follow-up, and PFS was calculated as the duration from the initiation of treatment to the objective progression of disease. Survival curves were created using the Kaplan-Meier method, and differences between the groups were evaluated using the log-rank test. All statistical tests were two-sided, $\mathrm{P}<0.05$ was considered to indicate a statistically significant difference and $95 \%$ confidence intervals were calculated. All statistical analyses were performed using EZR (Saitama Medical Center, Jichi Medical University, Saitama, Japan), which is a graphical user interface for $\mathrm{R}$ version 2.13.0 (The R Foundation for Statistical Computing). More precisely, it is a modified version of R Commander (version 1.6-3), designed to add the statistical functions frequently used in biostatistics.

\section{Results}

Patients. The clinical characteristics of the patients involved in this study are listed in Table I. A total of 67 patients were enrolled, with a median age of 68 years (range, $44-85$ years); $18 \%$ were female and $82 \%$ were male. The percentage of patients with adenocarcinoma was $58 \%$, and with squamous carcinoma was $31 \%$. The remaining $11 \%$ of patients had an undetermined diagnosis of adenocarcinoma or squamous carcinoma, or mixed characteristics of each. The proportion of patients with stage III disease was $48 \%$, and of those with stage IV disease was $42 \%$, at the stage of diagnosis. EGFR mutation status was evaluated in all patients with adenocarcinoma, $6 \%$ of whom were identified as having an exon 19 deletion, and $3 \%$ of whom had L858R in exon 21. Overall, $22 \%$ of patients had received one prior regimen and $78 \%$ had received $>2$ regimens. Treatment was imitated once the diagnosis was confirmed; platinum-based doublet therapy constituted the first-line therapy, and docetaxel or pemetrexed monotherapy followed as a second line therapy. If patients had an EGFR gene mutation, they were treated with an EGFR-TKI. The median treatment duration was 4.1 months (range, 1.9-19.2 months). The median follow up duration was 8.7 months (range, 2.3-30.0 months).

Response and survival. Treatment efficacy is summarized in Fig. 1. In total, $48 \%$ of patients manifested a partial response (PR) but none exhibited a complete response (CR); $27 \%$ of patients had stable disease (SD). The median PFS was determined to be 4.8 months (range, 3.8-5.6 months; Fig. 2A), and the median OS was 18.2 months (minimum, 10.4 months; Fig. 2B). According to histological analysis, the median PFS was 5.1 months for patients with non-squamous carcinoma (range, 4.0-6.7 months) and 3.8 months for patients with squamous carcinoma (range, 3.4-5.6 months; $\mathrm{P}=0.546$; Fig. 2C). The median PFS was 4.0 months for nab-PTX administered as second-line chemotherapy (range, 2.1-9.2 months) and 4.9 months as a third-line or later treatment (range, 3.8-5.8 months; $\mathrm{P}=0.541$; Fig. 2D).

Frequency of adverse events. The frequencies of adverse events (AEs) are detailed in Table II. In the patient cohort studied, the most common nab-PTX-associated AE (grade $\leq 3$ ) was neutropenia (29.9\%), followed by interstitial lung disease $(6 \%)$, infection $(6 \%)$ and fatigue $(6 \%)$. There was no fatal case recorded in the present study that was associated with AEs. However, $39.4 \%$ of patients were required to receive reduce doses or cease treatment due to AEs.

\section{Discussion}

This retrospective clinical study aimed to evaluate the effect and toxicity of a single-agent nab-PTX in patients with platinum-resistant NSCLC. The findings revealed that single agent nab-PTX was associated with an acceptable toxicity and a favorable response. In this study, $78 \%$ of patients were treated after receiving second-line therapy, as single-agent nab-PTX regimen has only recently been developed, thus was clinically available as a second-line therapy only to $22 \%$ of the current patient cohort. A comparison of our data with those of prior studies indicated that ORR was marginally improved in the present study, although there were more patients receiving subsequent therapies who were included in the present study. There was no statistical difference in PFS between those patients receiving second-line therapy and after a second-line regimen. History of prior taxane treatment was not associated with the PFS (data not presented). However, in the current study cohort, the rate of prior taxane use was $16 \%$, which was relatively low. Therefore, the actual resistance to taxane could be less, and the ORR may, therefore, be an improvement on other previous studies. The PFS and OS in this study were similar to those recorded in previous studies. According to histological analysis, the PFS of patients with non-squamous carcinoma was increased when compared with patients with squamous carcinoma; although this was not a statistically significant difference, it was consistent with the results of a previous study (8).

The optimal regimen for single-agent nab-PTX therapy has not yet been established. A dose-finding study involving patients with advanced NSCLC revealed that weekly nab-PTX 
Table I. Patients' characteristics.

\begin{tabular}{|c|c|}
\hline Categories & $\mathrm{n}, \%$ \\
\hline $\mathrm{n}$ & 67 \\
\hline Median age, years (range) & $68(44-85)$ \\
\hline \multicolumn{2}{|l|}{$\operatorname{Sex}(\%)$} \\
\hline Male & 82 \\
\hline Female & 18 \\
\hline \multicolumn{2}{|l|}{ ECOG PS (\%) } \\
\hline $0-1$ & 79 \\
\hline 2 & 21 \\
\hline \multicolumn{2}{|l|}{ Histology (\%) } \\
\hline Adenocarcinoma & 58 \\
\hline Squamous carcinoma & 31 \\
\hline Others & 11 \\
\hline \multicolumn{2}{|l|}{ Stage $(\%)$} \\
\hline I & 6 \\
\hline II & 4 \\
\hline III & 48 \\
\hline IV & 42 \\
\hline Postoperative recurrence $(\%)$ & 13 \\
\hline \multicolumn{2}{|l|}{ EGFR mutation status (\%) } \\
\hline Exon 19 deletion & 6 \\
\hline L858R in exon 21 & 3 \\
\hline \multicolumn{2}{|l|}{ Smorking history (\%) } \\
\hline Smorker & 84 \\
\hline Non-smorker & 16 \\
\hline \multicolumn{2}{|l|}{ Number of prior regimens $(\%)$} \\
\hline 1 & 22 \\
\hline$\leq 2$ & 78 \\
\hline \multicolumn{2}{|l|}{ Previous taxane $(\%)$} \\
\hline Yes & 16 \\
\hline No & 84 \\
\hline Median Treatment durations, months (range) & $4.1(1.0-19.2)$ \\
\hline Median Treatment cycles, $\mathrm{n}$ (range) & $3.6(1.0-13.6)$ \\
\hline
\end{tabular}

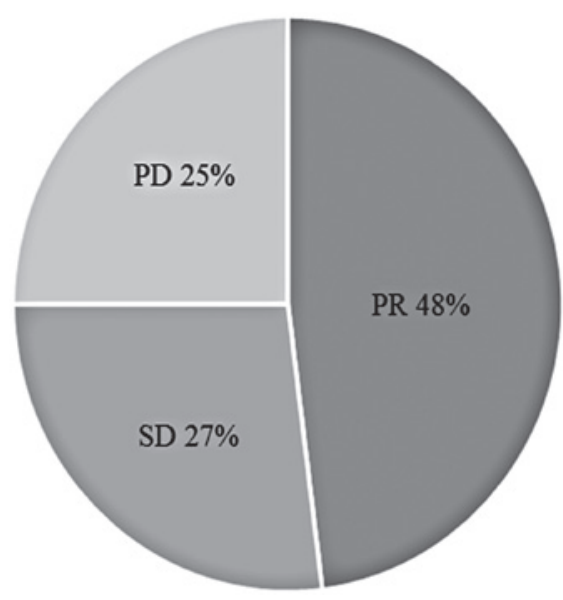

Figure 1. Patient's response rate. None patients exhibited CR. $48 \%$ of patients had a PR, and $27 \%$ had a SD. Furthermore, $25 \%$ of patients exhibited PD. CR, complete response; $\mathrm{PR}$, partial response; $\mathrm{SD}$, stable disease; $\mathrm{PD}$, progressive disease. $\left(100 \mathrm{mg} / \mathrm{m}^{2}\right)$ plus carboplatin administered at an area under the curve (AUC) of 6 every three weeks provided the greatest clinical benefit-risk ratio among the various weekly and three-weekly regimens examined (11). Furthermore, a multicenter phase III study evaluated the safety and efficacy of $100 \mathrm{mg} / \mathrm{m}^{2}$ nab-PTX followed by carboplatin AUC $6 \mathrm{mg} / \mathrm{ml} / \mathrm{min}$ (3). Therefore, in Kansai Medical University Hospital, a schedule of single-agent nab-PTX $100 \mathrm{mg} / \mathrm{m}^{2}$ on day 1,8 and 15 of a 21-day cycle was adopted.

For comparison with previous studies, the incidence of neutropenia and severe interstitial lung disease (ILD) was recorded as higher in the present study. A total of $78 \%$ of patients received $\geq 2$ therapeutic regimens; therefore, the recovery of bone marrow must have been delayed. Thus, the incidence of infection was also higher than previous studies. Although the safety of nab-PTX for ILD is not yet clear, it is generally considered that nab-PTX is as safe for ILD as is paclitaxel (3-4). In the current study, there were four patients who developed severe ILD, none of whom had a history of ILD. All patients who developed ILD were male and a smoker, though these associations were not statistically analyzed in the current study.

The National Comprehensive Cancer Network guidelines (https://www.nccn.org/professionals/physician_ gls/pdf/nscl.pdf) authorize the use of certain regimens for subsequent treatments: Docetaxel (DTX), pemetrexed, erlotinib, gemcitabine, ramucirumab plus DTX and nivolumab. Nab-PTX has not yet been listed. Among the recommended treatments, DTX is part of the same taxane class as nab-PTX, and has been previously used. Although the two agents have not been directly compared, it appears that the survival rates conferred by nab-PTX are comparable to those associated with DTX; however, nab-PTX is less toxic and is associated with fewer AEs. In the clinical trial of DTX as a monotherapy in patients with platinum-resistant NSCLC (12), 67-86\% of patients were noted to have developed grade $3 / 4$ hematologic toxicities. Furthermore, $22.4 \%$ of patients developed febrile neutropenia (FN). By contrast, in the phase II trial of nab-PTX as a monotherapy (8), only $4.5-19.5 \%$ of patients developed grade $3 / 4$ hematologic toxicities, none of which were FN. The PR was determined to be $33.7 \%$ in the DTX group and $31.7 \%$ in the nab-PTX group, respectively. In the separate clinical trials of DTX and nab-PTX, the patients' ethnic and demographical backgrounds were similar. Although each study was independent and simple comparison is challenging, it is speculated that nab-PTX may be less toxic. Furthermore, if ILD or edematous tendencies are present, DTX is problematic to use. Nab-PTX is considered to be relatively safe to use even in cases with ILD and renal dysfunction $(3,4,13,14)$. In the present study, $22 \%$ of patients were treated with nab-PTX as a second-line therapy. It was assumed that, if nab-PTX was selected as a second-line therapy for those patients it meant those patients may have experienced complications such as ILD or renal dysfunction, thus they could not receive the agents that are recommended for second-line regimens. Therefore, nab-PTX may be an alternative to DTX in the future. Randomized phase III clinical trials are required in order to compare the efficacy of nab-PTX and DTX as a subsequent treatment.

The current study had certain limitations, including that it was performed retrospectively, had a small sample size and involved only a single treatment center in Japan. 
Table II. Toxicities of Nab-PTX presenting as percentage of patients with adverse events.

\begin{tabular}{lccccc}
\hline & \multicolumn{5}{c}{ NCI-CTC grade (\%) } \\
\cline { 2 - 5 } Toxicity & Grade 1 & Grade 2 & Grade 3 & Grade 4 & Grade 5 \\
\hline Neutropenia & 6.0 & 10.4 & 23.9 & 6.0 & 0 \\
Anemia & 9.0 & 30.0 & 0 & 0 & 0 \\
Thrombocytopenia & 3.0 & 1.5 & 1.5 & 0 & 0 \\
Febrile neutropenia & - & - & 1.5 & 1.5 & 0 \\
Fatigue & 32.8 & 9.0 & 4.5 & 1.5 & 0 \\
Anorexia & 19.4 & 3.0 & 0 & 0 & 0 \\
Nausea & 11.9 & 0 & 1.5 & 0 & 0 \\
Constipation & 46.2 & 3.0 & 1.5 & 0 & 0 \\
Diarrhea & 1.5 & 0 & 0 & 0 & 0 \\
Myalgia/arthralgia & 19.4 & 0 & 0 & 0 & 0 \\
Peripheral sensory neuropathy & 31.3 & 16.4 & 1.5 & 0 & 0 \\
Dysgeusia & 13.4 & 0 & 0 & 0 & 0 \\
Infection & 3.0 & 3.0 & 1.5 & 4.5 & 1.5 \\
Interstitial lung disease & 0 & 0 & 4.5 & & 0 \\
\hline
\end{tabular}
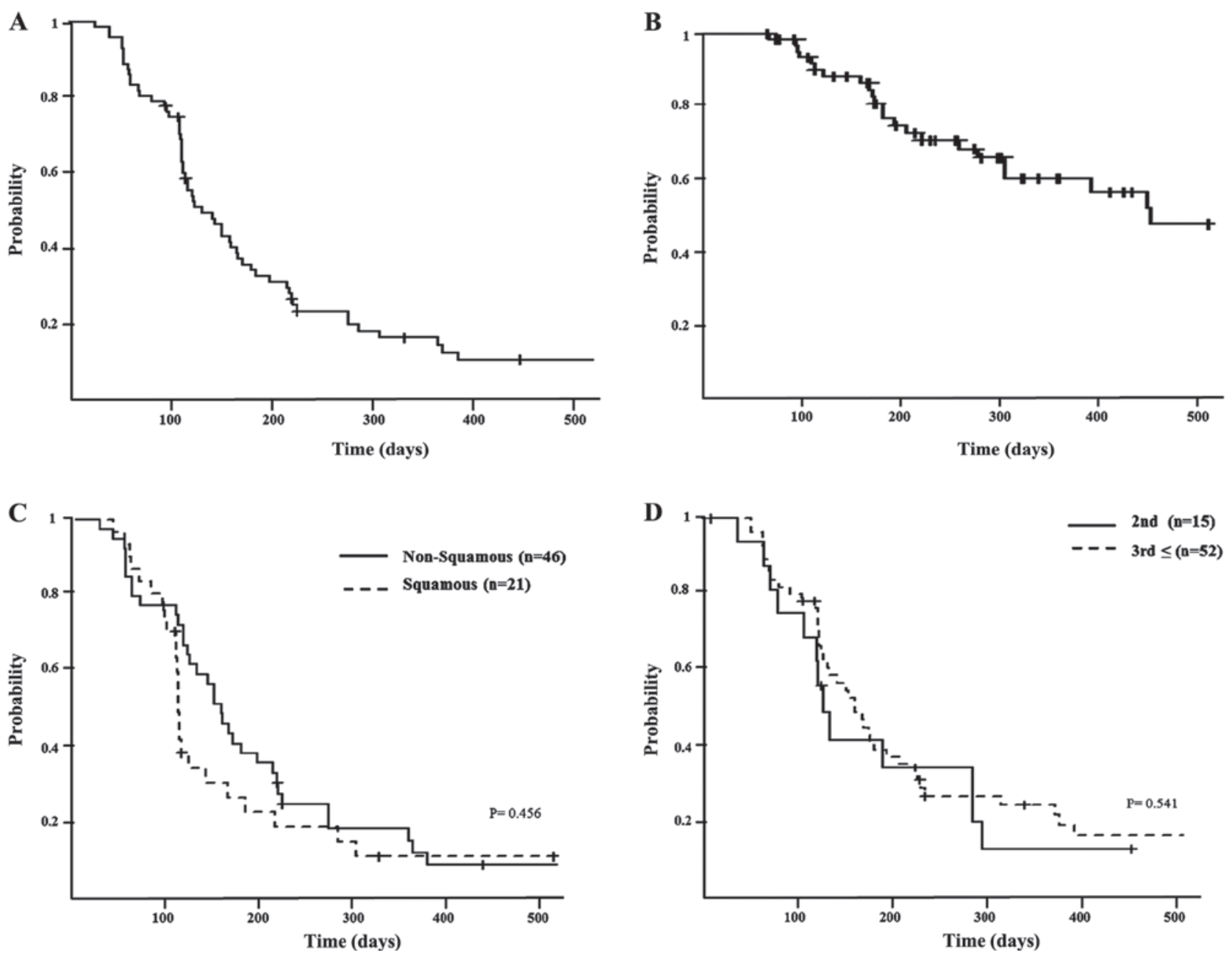

Figure 2. (A) Kaplan-Meier graphs for PFS. Median PFS was 4.8 months (range, 3.8-5.6 months). (B) Kaplan-Meier graphs for OS. Median OS was 18.2 months (range, 10.4 months; data not presented). (C) Kaplan-Meier graphs for the PFS of patients with advanced non-squamous cell carcinoma and squamous carcinoma. The median PFS for non-squamous was 5.1 months (range, 4.0-6.7 months), and for squamous it was 3.8 months (range, 3.4-5.6 months). There was no statistically significant difference ( $\mathrm{P}=0.456$ ). (D) Kaplan-Meier graphs for the PFS of patients with second-line and post-second-line therapy. The median PFS for second-line was 4.0 months (range, 2.1-9.2 months), and for post-second-line this was 4.9 months (range, 3.8-5.8 months). There was no statistically significant difference $(\mathrm{P}=0.541)$. $\mathrm{PFS}$, progression-free survival; OS, overall survival. 
In conclusion, the results of the present study indicated a clinically equivalent efficacy and safety for weekly-administered single-agent nab-PTX $100 \mathrm{mg} / \mathrm{m}^{2}$ on days 1,8 and 15 every 21 days in patients with platinum-resistant NSCLC. However, the safety profile for ILD must be validated. To assess the toxicity and efficacy of single agent nab-PTX in advanced NSCLC, the results of an ongoing clinical trial are expected.

\section{References}

1. Strauss GM, Herndon JE II, Maddaus MA, Johnstone DW, Johnson EA, Harpole DH, Gillenwater HH, Watson DM, Sugarbaker DJ, Schilsky RL, et al: Adjuvant paclitaxel plus carboplatin compared with observation in stage IB non-small-cell lung cancer: CALGB 9633 with the Cancer and Leukemia Group B, Radiation Therapy Oncology Group and North Central Cancer Treatment Group Study Groups. J Clin Oncol 26: 5043-5051, 2008.

2. Gradishar WJ, Tjulandin S, Davidson N, Shaw H, Desai N, Bhar P, Hawkins M and O'Shaughnessy J: Phase III trial of nanoparticle albumin-bound paclitaxel compared with polyethylated castor oil-based paclitaxel in women with breast cancer. J Clin Oncol 23: 7794-7803, 2005.

3. Socinski MA, Bondarenko I, Karaseva NA, Makhson AM, Vynnychenko I, Okamoto I, Hon JK, Hirsh V, Bhar P, Zhang $\mathrm{H}$, et al: Weekly nab-paclitaxel in combination with carboplatin versus solvent-based paclitaxel plus carboplatin as first-line therapy in patients with advanced non-small-cell lung cancer: Final results of a phase III trial. J Clin Oncol 30: 2055-2062, 2012.

4. Socinski MA, Okamoto I, Hon JK, Hirsh V, Dakhil SR, Page RD, Orsini J, Yamamoto N, Zhang H and Renschler MF: Safety and efficacy analysis by histology of weekly nab-paclitaxel in combination with carboplatin as first-line therapy in patients with advanced non-small-cell lung cancer. Ann Oncol 24: 2390-2396, 2013.

5. Fang Y, Wang L, Xia GH and Shi MQ: Clinical investigation of efficacy of albumin bound paclitaxel plus platinum compounds as first-line chemotherapy for stage III/IV squamous non-small cell lung cancer. Asian Pac J Cancer Prev 15: 7453-7, 2014.

6. Rizvi NA, Riely GJ, Azzoli CG, Miller VA, Ng KK, Fiore J, Chia G, Brower M, Heelan R, Hawkins MJ and Kris MG: Phase I/II trial of weekly intravenous $130-\mathrm{nm}$ albumin-bound paclitaxel as initial chemotherapy in patients with stage IV non-small-cell lung cancer. J Clin Oncol 26: 639-643, 2008.

7. Hu W and Zhang Z: A phase II clinical study of using nab-paclitaxel as second-line chemotherapy for Chinese patients with advanced non-small cell lung cancer. Med Oncol 32: 498, 2015.

8. Sakata S, Saeki S, Okamoto I, Otsubo K, Komiya K, Morinaga R, Yoneshima Y, Koga Y, Enokizu A, Kishi H, et al: Phase II trial of weekly nab-paclitaxel for previously treated advanced non-small cell lung cancer: Kumamoto thoracic oncology study group (KTOSG) trial 1301. Lung Cancer 99: 41-45, 2016.
9. American Joint Committee on Cancer: Lung cancer staging. 7th edition. https://cancerstaging.org/references-tools/quickreferen ces/Documents/LungMedium.pdf\#search $=\% 27 \mathrm{TNM}+$ classification +7th+lung+canacer+AJCC\%27. Accessed August 19, 2017.

10. Eisenhauer EA, Therasse P, Bogaerts J, Schwartz LH, Sargent D, Ford R, Dancey J, Arbuck S, Gwyther S, Mooney M, et al: New response evaluation criteria in solid tumors: Revised RECIST guideline (version 1.1). Eur J Cancer 45: 228-247, 2009.

11. Socinski MA, Manikhas GM, Stroyakovsky DL, Makhson AN, Cheporov SV, Orlov SV, Yablonsky PK, Bhar P and Iglesias J: A dose finding study of weekly and every-3-week nab-Paclitaxel followed by carboplatin as first-line therapy in patients with advanced non-small cell lung cancer. J Thorac Oncol 5: 852-861, 2010.

12. Shepherd FA, Dancey J, Ramlau R, Mattson K, Gralla R, O'Rourke M, Levitan N, Gressot L, Vincent M, Burkes R, et al: Prospective randomized trial of docetaxel versus best supportive care in patients with non-small-cell lung cancer previously treated with platinum-based chemotherapy. J Clin Oncol 18: 2095-2103, 2000.

13. Yasuda K, Igishi T, Kawasaki Y, Yamamoto M, Kato K, Matsumoto S, Kotani M, Sako T, Shigeoka Y, Sugitani A, et al: Phase II trial of weekly paclitaxel in previously untreated advanced non-small-cell lung cancer. Oncology 65: 224-228, 2003.

14. Khan A,McNallyD, TutschkaPJ and Bilgrami S: Paclitaxel-induced acute bilateral pneumonitis. Ann Pharmacother 31: 1471-1474, 1997. 